\title{
Xenografías femeninas en la literatura catalana contemporánea: Laila Karrouch y Najat El Hachmi, integración e identidad
}

\author{
Feminine Xenographies in the Contemporary Catalan Literature: \\ Laila Karrouch and Najat El Hachmi, Integration and Identity
}

\author{
Diego Muñoz Carrobles \\ dmunoz@invi.uned.es
}

Resumen: El objetivo de este artículo es realizar un acercamiento a la escritura de dos autoras catalanas de origen marroquí, Laila Karrouch y Najat El Hachmi. Sus obras, xenografías femeninas, contienen numerosos elementos de interés tanto para la literatura como para los estudios culturales, puesto que en ellas se reúnen conceptos importantes como la integración, el bilingüismo, el sexismo o la identidad, sin los cuales no podríamos comprender la sociedad europea contemporánea.

Palabras clave: xenografías, Laila Karrouch, Najat El Hachmi, literatura catalana

\begin{abstract}
The aim of this paper is to carry out an approach to two Catalan authors of Moroccan descent, Laila Karrouch and Najat El Hachmi. Their literary work, female xenographies, contain numerous elements of interest for both literary and cultural studies, since they deal with relevant elements such as integration, bilingualism, sexism or identity, without which we could not fully understand how present-day European society works.
\end{abstract}

Key words: xenographies, Laila Karrouch, Najat El Hachmi, Catalan literature

\section{INTRODUCCIÓN}

La inmigración constituye, sin lugar a dudas, uno de los fenómenos sociales más relevantes del siglo XX, especialmente agudizado desde su segunda parte, después de la Segunda Guerra Mundial y con los procesos de descolonización, cuando miles de personas abandonaron sus pueblos, ciudades y países a fin de encontrar una vida mejor en otro lugar. La consecuencia más 
evidente de los procesos migratorios ha sido la reconfiguración de las sociedades de origen y de acogida, dando lugar, en el caso de éstas, a sociedades multiculturales, compuestas por grupos de religiones, idiomas, razas y costumbres diferentes.

Fruto de esta multiculturalidad han surgido las xenografías, entendidas como las escrituras producidas por autores migrantes en las sociedades de acogida y en el idioma de adopción. En este trabajo, además, nos ocuparemos de las xenografías femeninas, mediante el análisis de las obras de Laila Karrouch y de Najat El Hachmi, nacidas en el norte de Marruecos pero instaladas en Cataluña desde mediados de los años 80 del siglo pasado y escritoras en lengua catalana. La literatura contemporánea le ha reservado un espacio cada vez más importante a las voces de mujeres que experimentan el exilio, la inmigración, la desterritorialización y que narran en sus xenografías el encuentro del Yo con el Otro. En palabras de Margarita Alfaro y Beatriz Mangada:

Las mujeres, más que nunca, en el momento histórico en el que vivimos a consecuencia del influjo de los movimientos migratorios económicos y los desplazamientos vinculados al exilio ideológico, cultural y religioso, se han convertido en las transmisoras de un nuevo mundo que está por construir y en el que desempeñan un papel protagonista. ( Alfaro y Mangada 2014: 10)

La sociedad de acogida para estas dos autoras ha sido Cataluña, que, desde la Revolución Industrial de la segunda mitad del siglo XIX, se ha erigido en polo de atracción de inmigrantes y tierra de oportunidades para miles de trabajadores que se desplazaron desde otras partes de España durante la dictadura franquista (principalmente Andalucía y Extremadura) o desde latitudes más lejanas, desde los años 80 y hasta la actualidad. Según las cifras del Instituto Nacional de Estadística referidas a 2016, había en Cataluña más de un millón de ciudadanos de origen extranjero, de los cuales doscientos mil, aproximadamente, eran marroquíes ${ }^{1}$.

\begin{tabular}{|l|r|}
\hline Población total extranjera & 1.020 .631 \\
\hline Población marroquí & 210.838 \\
\hline
\end{tabular}

Nuestra intención, a lo largo de estas páginas, será ver cómo funcionan en las obras de Laila Karrouch y de Najat El Hachmi dos conceptos clave de los estudios culturales, la integración y la identidad, en las distintas dimensiones que atañen a estas dos autoras catalanas de origen marroquí: la influencia de la religión musulmana en los procesos de aculturación y de acogida, la pertenencia a comunidades linguiísticas alóctonas instaladas en una sociedad bilingüe $y$, por último, la relevancia del género, es decir, además de la lengua y la religión, cómo se ha reflejado en sus escrituras el hecho de ser mujer.

\footnotetext{
${ }^{1}$ http://www.ine.es/jaxi/Datos.htm?path=/t20/e245/p04/provi/10/\&file=0ccaa002.px
} 


\section{PUNTOS EN COMÚN ENTRE LAILA KARROUCH Y NAJAT EL HACHMI}

Las dos autoras que analizaremos en este trabajo presentan numerosas similitudes, tanto en su trayectoria vital como literaria. En primer lugar, ambas autoras nacieron en la provincia marroquí de Nador, en el norte del país, cerca de la ciudad española de Melilla. Laila Karrouch (1977) y Najat El Hachmi (1979) son casi coetáneas y llegaron a Cataluña a mediados de los 80, ambas se instalaron en la ciudad de Vic, cabecera de la comarca de Osona, a unos 70 kilómetros de Barcelona. Una de las características comunes de su trayectoria vital será, por ejemplo, pertenecer a la llamada «Generación 1b» de la inmigración, esto es, niñas nacidas en el país de origen pero emigradas a temprana edad y escolarizadas en el país de acogida. Tanto Karrouch como El Hachmi ocupan, pues, ese espacio intermedio entre la primera y la segunda generación de inmigrantes y esta será una de las claves de su obra literaria, la intermediaridad.

La región marroquí en torno a Nador conforma, al igual que Cataluña, un espacio plurilingüe donde conviven diferentes idiomas y tradiciones culturales. $\mathrm{Al}$ amazigh o bereber se le une el árabe marroquí o dariya, el árabe clásico de la religión, el francés, lengua de las elites o incluso, el español. Las niñas Laila y Najat desembarcan en Vic, localidad eminentemente catalanoparlante, pero donde también se habla español y serán, como ya iremos desgranando en estas páginas, especialmente sensibles a la coexistencia de diferentes lenguas y costumbres, cuya presencia y suma son tratadas de manera positiva.

Tanto Laila Karrouch como Najat El Hachmi poseen trayectorias literarias muy parecidas. Al principio de sus carreras literarias exploraron la veta autobiográfica, con obras que recrean o directamente describen su experiencia migratoria y las distintas vicisitudes que han tenido que vivir en el proceso de adaptación a la sociedad de acogida. A esta primera etapa pertenecen el ensayo autobiográfico Jo també sóc catalana (2004) de Najat El Hachmi y la novela De Nador a Vic (2004) de Laila Karrouch, destinada al público juvenil ${ }^{2}$ y en la que se narran episodios extraídos de la propia experiencia de la autora.

Podríamos hablar de una segunda etapa, durante la cual no se abandona el «exotismo» de la experiencia migratoria o de la pertenencia a una comunidad inmigrante, pero se reelabora y literaturiza, dando lugar a la novela que consagró a El Hachmi, L'últim patriarca (2008), con la que obtuvo el premio Ramon Llull, uno de los más prestigiosos en lengua catalana y en la cual recrea la vida de una niña que emigra desde Marruecos hasta una pequeña ciudad del interior de Cataluña y que deberá enfrentarse a su padre para poder salir adelante. Dentro de esta etapa podemos incluir también la novela Petjades de Nador (2013) de Laila Karrouch, con tintes autobiográficos y que insiste en la temática migratoria, pero destinada a un público adulto.

Tras estas dos etapas, que están marcadas por el origen geográfico de las autoras, era de esperar una tercera etapa de expresión literaria propia y liberada

\footnotetext{
${ }^{2}$ Obra ganadora del Premi Columna Jove 2004.
} 
de las raíces culturales, que podemos atisbar en la obra La caçadora de cossos (2011) de Najat El Hachmi, donde de abandonan los motivos relacionados con la experiencia migratoria para explorar nuevos horizontes literarios, en este caso, la historia de una mujer y sus distintos amantes. Sin embargo, la propia autora volvió a la segunda etapa con La filla estrangera (2015), una obra centrada en la experiencia de la hija inmigrante que se rebela frente a la figura materna, con la cual mantiene serias diferencias, fruto del proceso de integración en el país de acogida y que pone en evidencia la posición más conservadora de la madre. Laila Karrouch, por su parte, ha regresado a la novela juvenil con Quan a l'Isma se li van creuar els cables (2015), en la que narra las vivencias de un adolescente de origen marroquí.

\section{LA INMIGRACIÓN MARROQUÍ EN CATALUÑA}

Tal y como se ha mencionado anteriormente, Cataluña ha sido históricamente una sociedad receptora de inmigración. No se trata de un fenómeno reciente, sino que se remonta a los primeros años del siglo XX, situación retratada por Eduardo Mendoza en La ciudad de los prodigios (1986), con la llegada de mano de obra para la Exposición Universal de Barcelona. De este asunto se ocuparía posteriormente Francesc Candel en su obra Els altres catalans (1964), imprescindible para quienes quieran estudiar el fenómeno migratorio «charnego» lejos de números y estadísticas: «Els andalusos hem ocupat Catalunya sense disparar ni un sol tret» (2008: 171). El desarrollo industrial y económico de Cataluña la convirtieron en lugar de destino para cientos de miles de trabajadores llegados desde otros puntos de España, principalmente Andalucía, Murcia, La Mancha o Extremadura, que se instalaron sobre todo en el cinturón metropolitano de Barcelona. Su fuerza demográfica fue tal que la candidatura del Partido Socialista Andaluz llegó a obtener representación en el Parlament de Catalunya en las elecciones de 1980.

Sin embargo, los 80 marcan un antes y un después en la configuración de las migraciones hacia tierras catalanas. Mientras que pierde fuerza la inmigración procedente de otras zonas del Estado, la ganan las comunidades migrantes procedentes del Magreb y América Latina, en un primer momento, y de Europa del Este y Asia, posteriormente. Se trata de mano de obra «con un nivel medio de formación y [que] se emplean en el servicio doméstico, la construcción o la agricultura como peones.» (Solé 2000: 216). Es el caso de los padres de Laila Karrouch y de Najat El Hachmi, que consiguen encontrar trabajo en la comarca de Osona, con una fuerza presencia de la industria agroalimentaria, a mediados de la década de 1980.

Las páginas escritas por nuestras dos autoras evidencian las dificultades experimentadas por sus familias a la hora de instalarse en Cataluña, ya que en ocasiones experimentarán la hostilidad e incluso el rechazo de los de «dentro» hacia quienes vienen de «fuera»:

Parlaven d'immigrants i jo ni tan sols coneixia la paraula. [...] O sigui que érem nosaltres, els immigrants, Però jo no acabava de sentir-m'hi identificada, 
¿què volia dir que ara ens diguessin immigrants, deixàvem de ser els mateixos o només teníem un nou nom? Els pares catalans no volien portar els seus fills a les escoles dels immigrants... (El Hachmi 2004: 77-78)

La niña Najat no comprende por qué ella y los suyos han adoptado una nueva identidad mediante una denominación, una etiqueta, la de inmigrantes, que ella no conocía. Pero esta etiqueta cobrará una carga semántica aún mayor en el caso de pertenecer a una comunidad de religión musulmana, para algunas personas ellas ya no son Laila o Najat, sino «moras».

Un dia, plegant, a les dotze, una nena més petita que jo va acostar-se cap a mi i em va dir que la seva mare no la deixava jugar amb «mores». [...] En Sergi, que era testimoni de Jehovà, però que al principi no ho reconeixia, també em deia «mora». A vegades no volia que jugués amb ell. (Karrouch 2004: 45-46)

La sociedad española y, en particular, la catalana, no han conocido fenómenos sonados de islamofobia ni de racismo, sino episodios puntuales que, como la espuma de la cerveza, han terminado por disiparse. Nos referimos concretamente al caso del partido Plataforma per Catalunya (PxC), encabezado por Josep Anglada, que alcanzó sus mejores resultados en las elecciones municipales de 2011, obteniendo más de un $2 \%$ de los votos y consiguiendo más de sesenta concejales en distintos ayuntamientos de Cataluña. ${ }^{3}$ En Vic, donde el propio Anglada era candidato, PxC obtuvo casi el $20 \%$ de los votos y cinco de los veintiún concejales que compusieron la recién elegida corporación municipal en 2011.

La ideología de Anglada y los suyos se resume en el lema del partido: «Primero los de casa», que sintetiza en cuatro palabras el sentimiento de rechazo hacia aquellos que no son «de casa», el odio al «ellos» frente a la defensa del «nosotros», conceptos todos estos difíciles de aplicar y de delimitar más allá de los manifiestos electorales. El auge de la PxC se explica en un contexto de crisis económica incipiente que busca chivos expiatorios fáciles a través de mensajes simples, demagógicos, como la declaración de Josep Anglada poco después de su eclosión electoral: «Un moro siempre será un moro, nunca será ni español ni catalán» (El Mundo, 26/05/2011).

Diferentes voces se alzaron contra las ideas racistas y xenófobas de la Plataforma per Catalunya, entre ellas las de Laila Karrouch y Najat El Hachmi, afectadas en primera persona, y por partida doble, por la popularidad de este movimiento político, al ser marroquíes y de Vic.

Najat El Hachmi lamenta que l'actitud vers la immigració s'hagi endurit per culpa dels discursos de polítics com Anglada. «Els problemes de convivència de què parlaven aquestes forces polítiques no eren evidents, i en canvi amb

${ }^{3}$ Pese a parecer un porcentaje casi desdeñable, era preocupante el avance de esta formación en tan solo cuatro años, puesto que la PxC solo había obtenido un $0,43 \%$ del total de los sufragios emitidos en Cataluña en las elecciones locales de 2007. 
aquest foment del trencament social pot ser que anem a més», denuncia El Hachmi. «Són uns discursos que no són veritat, es fonamenten en moltes mentides» critica l'escriptora, que ha vist amb tristor com a Vic i a d'altres indrets de Catalunya les postures xenòfobes «tenen conseqüències en la convivència». (ACN 2011)

Així, Josep Anglada serà el proper alcalde de Vic? Espero que no. Ara: si guanya, guanya. Si la gent el tria, se l'haurà de respectar. Però si llavors vol que les dones musulmanes $-\mathrm{O}$ «mores», com diu ell- es treguin el mocador, jo tinc clar què faré: no me'n posaré un, no. Me'n posaré dos! Només faltaria que una persona hagi de decidir les coses privades de la gent. (Ferrer 2013)

Algunas actitudes racistas y xenófobas aparecen en las páginas escritas por estas dos autoras y corresponden, por ejemplo, a la reticencia a alquilar pisos a marroquís o a criticar el uso del velo por parte de algunas mujeres musulmanas. Se trata de una xenofobia más pasiva que activa, es decir, no hay ataques directos sino silenciosos, que pueden llegar a minar la autoestima del individuo inmigrante y empujarlo hacia su asimilación en la sociedad de acogida, es decir, que privilegie los valores culturales del país de destino frente a la cultura de origen, que se percibe como un obstáculo. Así narra Najat El Hachmi uno de estos episodios cotidianos, cargados de injusticia, en La filla estrangera:

He anat a veure un pis $i$ ho he fet sola. No puc portar la mare perquè en veurela amb mi, amb el mocador, els que lloguen pisos solen canviar d'opinió. Solen canviar d'opinió de totes maneres, però quan hi vaig amb la mare ja queda clar des del primer moment que no tenim cap possibilitat que considerin agafar-nos de llogateres. Al començament m'indignava i m'agafaven ganes de cridar, d'acusar-los de racistes (El Hachmi 2015: 84)

Con todo, la imagen de los marroquís en las obras de El Hachmi y de Karrouch dista de la idealización. El lector puede observar frecuentemente cómo la escritura de estas dos autoras huye del paternalismo y nos presenta individuos -generalmente hombres- que no cumplen con los preceptos del islam y que viven el drama de una inadaptación social que trasladan a su vida doméstica, como bien refleja Laila Karrouch en el personaje del marido, en Petjades de Nador, o bien El Hachmi en L'últim patriarca, cuyo protagonista, llamado Mimoun, encarna al perfecto villano: esposo infiel, padre despótico y agresivo, mal musulmán:

El pare devia obrir una cervesa darrere l'altra mentre parlava cridant amb la mare; els meus germans no es van despertar i cap d'ells no ha sabut mai què va passar aquella nit. Jo vaig sentir que cridava i no vaig saber què fer. [...] La mare va dir: ves-te'n, com va poder, però jo la podia veure, amb el coll estirat i el ganivet que ja li tocava la pell. [...] Vine, filla, vine, que veuràs com degollo la teva mare. Ho vols veure? (El Hachmi 2008: 174-175)

De hecho la propia autora habla de doble moral en el islam, a propósito de este personaje, el padre de la protagonista, algo que es aplicable, en su opinión, al resto de países musulmanes: 
És un personatge molt contradictori amb la doble moral típica que també existeix molt als països àrabs. [...] Això és així, és el dia a dia. Fa alguns anys recordo que vaig anar a Casablanca i el debat era si al Codi de circulació es prohibia conduir begut, quan a l'islam és prohibit beure, però s'ho havien de plantejar perquè hi havia tants accidents de trànsit per la beguda que no tenien altre remei... La doble moral és constant i et confon. (Bacardit 2015)

En general, la visión de la religión dentro de la comunidad inmigrante que nos ofrecen nuestras autoras coincide con el esquema general de la aculturación, es decir, las primeras generaciones conservan intactas fe, tradiciones y ritos mientras que las generaciones posteriores se van secularizando, lo cual provocará fricciones dentro de los núcleos familiares en torno a temas como el divorcio, la emancipación o el velo islámico, según veremos más adelante. Si bien se dan casos asimilacionistas, como el que describe Laila Karrouch, cuando su madre se opone a que ella siga el ayuno del mes de Ramadán para que pueda seguir compitiendo en atletismo, algo al que la niña Laila se opone: «Era molt important practicar el Ramadà. Al Marroc la mare no m'ho hauria prohibit, n'estava segura.» (Karrouch 2004: 55).

\section{LENGUA E INMIGRACIÓN: LA FRONTERA INVISIBLE}

Tradicionalmente, el español había sido la lengua de la inmigración en Cataluña, la lengua de los llegados de fuera, frente al catalán como lengua intragrupal de la comunidad de acogida. En numerosas ocasiones, tanto Karrouch como El Hachmi describen escenas cotidianas en las cuales los habitantes de Vic, en tiendas u otros establecimientos, se dirigen a los personajes marroquíes en español, dando por supuesto que, por ejemplo, una chica de aspecto extranjero es incapaz de expresarse en catalán, lo cual provoca un cierto malestar, un sentimiento de herida que no se cierra:

Entrem en una botiga antiga, de tota la vida, mig ancorada en el passat. [...]
És el meu torn: -Dime, ¿qué quieres? -De sobte el senyor Eladi, tan atent, ja
no ho és tant amb mi. I aquesta mania de parlar-me en castellà, ell no s'ho deu
pas imaginar que amb les seves paraules ja m'ha tornat a posar el dit en aque-
lla llaga que mai es cura, perquè un o altre cada dia t'hi furga. Potser ho fa
endut per la bona fe, és clar que tots els immigrants deuen saber només caste-
llà. (El Hachmi 2004: 50)

La realidad lingüística en Cataluña ha cambiado desde la entrada en vigor del primer Estatut d'Autonomia en 1981 y de las primeras medidas políticas a favor de la lengua catalana, como la Llei de Normalització Lingüística de 1983, gracias a la cual, el catalán entra en el sistema educativo. Así pues, la Cataluña a la que llegan las niñas Laila y Najat es una sociedad bilingüe pero cuya enseñanza pública se realiza en lengua catalana. Ya no habrá obstáculos para que las segundas generaciones de inmigrantes aprendan catalán e intenten cruzar esta frontera invisible que había separado a la comunidad de origen de las comunidades inmigrantes. 
La aparición de estas xenografías significa, en nuestra opinión, el éxito de la política lingüística catalana en materia de inmigración, puesto que distintos autores de origen extranjero han entrado a formar parte del sistema literario gracias a su escolarización en catalán. La industria editorial se ha ocupado de promocionarlos e incluso, de premiarlos. Ahora bien, el bilingüismo que podemos leer en algunas de las obras de Laila Karrouch y de Najat El Hachmi no está exento de problemas ni de situaciones incómodas, sobre todo al principio, «El pare deia que feia una sopa de català-castellà i reconec que m'empipava una mica perquè la Hayat i en Nourdine se'n reien» (Karrouch 2004: 42) e incluso, traumáticas, como el momento en que la niña protagonista de L'últim patriarca debe acompañar a su madre al ginecólogo y ejercer de intérprete, escena en la que volvemos a ver cómo esta autora se aleja de una visión idealizada del bilingüismo:

Procurava d'estar darrere la cortina mentre traduïa el que anava dient la llevadora. Hi havia coses que no sabia passar d'un idioma a l'altre, que no volia passar d'un idioma a l'altre. Quina va ser l'última vegada que li va venir la regla a la mare? [...] Déu, Déu, volia fugir corrents de tot allò, jo no les vull saber, totes aquestes coses, i encara menys, traduir-les a un idioma on no hi havia cap paraula que jo conegués per a relacions sexuals que no fossin paraulotes. (El Hachmi 2008: 216-217)

Otro ejemplo de conflicto relacionado con el bilingüismo se refiere a la doble identidad que se forma dentro de la persona bilingüe. Del mismo modo en que Laila Karrouch se refería a la «sopa», a la mezcla de usos lingüísticos, las identidades también se superponen o se amalgaman y dan lugar a episodios como el narrado por Najat El Hachmi en Jo també sóc catalana (2004), cuando gana un concurso literario en la escuela:

L'endemà de recollir el premi, el diari comarcal em presentava en portada, amb fotografia i tot: «Jove marroquina guanya un premi en català». El titular em va decebre, volia que m'afalaguessin, és clar, un massatge a l'ego sempre va bé, però no per ser marroquina sinó per ser escriptora.[...] Fins i tot, una senyora d'un bar on ens coneixien de tota la vida em va dir: S'hi devien presentar molt pocs a aquell concurs, no? (El Hachmi 2004: 43-44)

¿Por qué es necesario justificar la elección de una lengua de uso cotidiano o de uso literario? El catalán es, para los personajes de las obras analizadas, una lengua tan «suya» como para los habitantes de Vic. A través de la escuela y de la vida en un entorno de habla catalana, la lengua catalana se ha convertido en vehículo de expresión de sentimientos y de realidades que no tienen nombre en la lengua materna. Sobre ello reflexiona la protagonista de La filla estrangera cuando visita su aldea natal antes de su matrimonio concertado:

Avui és el meu dia. La frase només té sentit en aquesta llengua que en aquests dies de vacances «al nostre país», «a la nostra terra», s'ha convertit en llengua dels meus pensaments, només me la sento per dins, Si tornés a viure aquí, 
quant de temps podria conservar-la? Quant de temps seguiria sent la meva llengua de pensament? (El Hachmi 2015: 47)

Vemos cómo, a lo largo de las carreras literarias de ambas autoras, tiene lugar un intento de desprenderse de la etiqueta «marroquí», no por vergüenza ni por auto odio, sino para reclamar su valor como autoras en lengua catalana. No se trata de un asunto baladí, sino que es un fenómeno al que se han enfrentado otros autores xenográficos, que se han esforzado por entrar en un sistema literario determinado por méritos propios, no solamente por el valor simbólico de su comunidad de origen ni por el significado social de su obra. No obstante, el hecho de seguir cultivando los temas relacionados con la inmigración en sus obras, bien por decisión personal, bien por motivos editoriales, supone, a nuestro juicio, una barrera a la hora de dejar atrás las etiquetas identitarias.

\section{ISLAM Y MUJER INMIGRANTE}

El último de los aspectos que queremos tratar en este trabajo se refiere a la imagen de la mujer musulmana inmigrante en la sociedad de acogida. Tanto Laila Karrouch como Najat El Hachmi, de nuevo, evitan adoptar una actitud paternalista y generalizadora hacia las mujeres de su propia comunidad etnolingüística: cada familia es un mundo y cada mujer, de primera o de segunda generación, será libre para tomar sus propias decisiones con respecto al mantenimiento de la tradición musulmana.

Uno de los temas recurrentes será el papel de la mujer como garante del orden y de la tradición dentro del núcleo familiar, esto es, la mujer marroquí inmigrante de primera generación se retrata como una persona que permanece en la casa, que se encarga de las tareas de la casa y del cuidado de los hijos y que, en ocasiones, se comporta de manera sumisa, pensemos en la mujer de Mimoun, en L'últim patriarca, que aguanta malos tratos, amenazas e incluso la humillación de tener que ir de vacaciones con la amante española del marido. O bien la madre del alter ego de Laila Karrouch en Petjades de Nador, quien, en su afán por mantener las costumbres aprendidas en Marruecos, llega al hospital donde trabajaba la hija y en el cual se encontraba ahora hospitalizada tras dar a luz, cargada de alimentos: «Totes ho hem fet així $i$ els nostres fills són sans $i$ forts. Aquestes coses no t'han de fer vergonya, Laila.» (Karrouch 2013: cap. X)

La madre de Laila menciona, de manera involuntaria, uno de los motivos por los cuales el estudio de las xenografías posee un valor no solo literario sino social. A menudo los autores ponen en boca de sus personajes conceptos, metáforas o reflexiones tanto o más interesantes que los que podemos extraer de obras académicas o de divulgación. Cuando la madre le dice a Laila que no debe tener vergüenza, nos encontramos ante uno de los conflictos clave a la hora de entender el fenómeno de la aculturación de las comunidades de inmigrantes. Frente a la tendencia asimiladora, que supone un rechazo de la cultura de origen, que produce «verguienza» porque es diferente o es percibida como inferior con respecto a la sociedad a la que se aspira a pertenecer, tenemos la 
tendencia integradora, que defiende que un individuo puede configurar la propia identidad con elementos de la cultura de origen y de la cultura receptora.

En el caso de la comunidad marroquí, la religión constituye uno de los rasgos definitorios principales, algo que «forma part del dia a dia, és un element quotidià» (Bacardit 2015), por ello, la pérdida del sentimiento religioso desencadena un conflicto de identidad social y personal en los miembros de las generaciones primera-b o segunda. Así lo glosa Najat El Hachmi, cuando en la adolescencia se pregunta por la existencia de Dios y, por ende, por la verdad o falsedad de muchos de los valores sobre los cuales reposa su cultura de origen:

Si Déu no existia, tot allò que tant havia estimat, les oracions, la veu del muetzí, els divendres a la mesquita, tot era una gran mentida ? Tot el que componia la meva identitat marroquina, el que m'havia diferenciat dels infidels, era perversament fals? No hi trobava cap sentit, esdevenia absurd, m'abocava precipitadament a l'abisme. (El Hachmi 2004: 113)

Y ese abismo que menciona Najat El Hachmi funciona como una poderosa metáfora del desarraigo y del sentimiento de no-pertenencia, una situación intermedia entre la cultura de origen y la de acogida, que se perciben como lejanas e inalcanzables. Otros «abismos» que deben sortear algunos personajes femeninos descritos por estas dos autoras se refieren a la emancipación de la mujer o al divorcio, todavía tabús en algunas comunidades inmigrantes. La mujer inmigrante musulmana, en cuanto garante de la tradición, como se apuntaba anteriormente, es a menudo estereotipada como conservadora, sumisa u obediente. Sin embargo, el contacto con la sociedad de acogida o bien la educación en un sistema de igualdad de derechos - al menos, en teoría- provoca que la mujer musulmana tome conciencia de su individualidad y desee tomar las riendas de su futuro. La esposa humillada de L'últim patriarca recibe continuos consejos de sus vecinas catalanas para que abandone al marido, pero ellas, a ojos de esta esposa y madre prototípica, no comprenden el peso de la tradición musulmana, una actitud que Najat El Hachmi ha descrito como «salvar a las moritas de los yugos de sus esposos» y que no resulta tan fácil como parece:

És que què els ofereixes, a canvi? Moltes vegades s'exigeix que [les dones musulmanes] facin aquest procés d'alliberament, però fer-lo és com estar agafada a una liana sense poder-te agafar a cap altra. Si tu no tens l'altra per poder-te agafar, no funciona. Es paga molt car: amb solitud, amb falta de suport... (Bacardit 2015)

De las obras que estamos analizando en este trabajo, la que más a fondo trata el conflicto personal de las mujeres marroquís de segunda generación es, a nuestro juicio, La filla estrangera, de Najat El Hachmi (2015). Según habíamos visto en la presentación de las autoras, El Hachmi «regresa» a la temática migratoria tras haber explorado nuevos caminos narrativos en su anterior novela La caçadora de cossos, donde la sexualidad femenina es el tema principal. La influencia de esta novela se deja ver a lo largo de las páginas de La filla estrangera, puesto que, frente a la «heroína» protagonista de L'últim patriarca, 
la «supermana» como ella misma se denomina, estamos ante una antiheroína, una mujer muy joven, que ha vivido con su madre en una pequeña ciudad «de purins» -trasunto de Vic- desde su llegada a Cataluña, pero que, tras conseguir una nota sobresaliente en selectividad, accede a abandonar los estudios para casarse en Marruecos con su propio primo, al que acabará detestando. De esa falta de independencia, nacerá el deseo de huida hacia la gran ciudad, sinónimo de libertad y donde las reglas de la tradición marroquí no tienen valor:

\begin{abstract}
Són tants els pensaments positius que arribo a imaginar que podria fer-ho tot, podria viure una vida més meva enmig de la ciutat i fer que la meva mare vingui amb mi, demanar-li que sigui ella la que s'adapti al que jo sé que em faria feliç. Per què no podem viure aquí, fer el que ens sembli i no haver de donar explicacions a ningú? No haver-me de casar amb ningú ni haver de seguir el camí que tinc traçat per un mandat més col-lectiu que diví. (El Hachmi 2015: 101-102)
\end{abstract}

Siguiendo el motivo de lo que Najat El Hachmi denomina acertadamente «mandato colectivo», podemos describir la actitud hostil de la familia de Laila en Petjades de Nador (2013) ante la posibilidad de divorcio de su hija. Esta idea se nos presenta como algo escandaloso, una traición a la familia, a la comunidad, una ruptura del orden establecido, no solo en Vic, sino también en Marruecos -no olvidemos el peso del lugar de origen, generalmente pueblos muy pequeños donde el concepto de honor y la reputación de la familia tienen un papel destacado en la sociología del lugar. La mujer musulmana debe aguantar, ser paciente, ofrecer la otra mejilla. Si el marido (Alí) ha sido infiel, se trata de un pequeño error sin importancia, la esposa ha de saber perdonar:

\begin{abstract}
Els pares es van presentar a casa amb els tiets, els germans del pare i un parell de senyores que van sortir de vés a saber on. L'Alí era el mig de tot; se'l veia petit, indefens, i notablement desmillorat. [...] -Filla, hem vingut a salvar aquest matrimoni. No és just el que estàs fent amb el teu marit. Ha tingut una petita relliscada, s'ha deixat enganyar pel xitan. Tots els matrimonis tenen problemes -va dir la mare. (Karrouch 2013: cap. XII)
\end{abstract}

No podía faltar en las obras analizadas el controvertido asunto del velo islámico. Ambas autoras piensan que se trata de un debate social estéril, un tema que determinados sectores de la sociedad se empeñan en desenterrar cada cierto tiempo. Sin embargo, las actitudes que los personajes femeninos de las obras de Laila Karrouch y de Najat El Hachmi adoptan frente al velo son variadas y van desde el rechazo por ser un símbolo de las raíces de las que quieren alejarse o bien la reafirmación de una cultura propia que no excluye la aceptación de las normas de convivencia de la sociedad de acogida. Y son precisamente los miembros de esta última quienes demuestran, e juicio de las autoras, una falta de conocimiento sobre esta prenda, a veces llamada «pañuelo» de manera despectiva, que se considera símbolo de la opresión, frente a la cual las mujeres musulmanas tendrían que rebelarse. Nos encontramos, de nuevo, frente al «yugo» que describíamos líneas arriba. 
Per què no es treuen el mocador? Era la pregunta del milió, una pregunta que et feia fins i tot la persona més culta que et poguessis imaginar. Aquella peça de roba que durant la meva infantesa havia sigut quelcom màgic que desplegava damunt del meu cap per notar-ne la frescor de la tela [...] Tot allò es convertia en tema polèmic dia sí dia també. (El Hachmi 2004: 155)

De esta manera, una prenda de ropa estigmatiza a su portadora, que en ocasiones intentará buscar un pretexto para no llevar velo o chilaba, tal vez con el fin de no parecer tan diferente o de asimilarse a través de la apariencia física:

La mare li deia al pare que quan anés amb nosaltres no es posaria la gel·laba ni el mocador perquè les mares d'altres companys li deien que estava més maca sense, Aquesta vegada el pare va acceptar la decisió de la mare. El pare va anar al mercat amb la mare i li va comprar un parell de faldilles, això sí, fins als genolls, i dues camises de conjunt. (Karrouch 2004: 59)

La impresión general que el lector extrae tras el análisis de estas obras es que las decisiones de las mujeres musulmanas necesitan justificarse constantemente, han de ser explicadas, razonadas y defendidas, algo que quizás no ocurriría ni en el caso de los hombres, ni de otra religión.

\section{A MODO DE CONCLUSIÓN: ESCRITORAS Y MUJERES «ENTRE DOS MUNDOS»}

En las palabras de nuestras dos autoras podemos encontrar una defensa de la idea de integración de la comunidad marroquí en la sociedad catalana. Se trataría, pues, del triunfo, de la integración, frente a las otras estrategias de aculturación: la asimilación, la segregación y la marginación. Integrarse consistirá, básicamente, en vivir entre dos mundos, entre dos culturas, enriqueciéndose gracias a los elementos positivos que ambas poseen, a pesar de los desafíos y de las dificultades que surgen del proceso migratorio: «Em vaig passar molt de temps intentant triar una de les dues nacionalitats $i$ em vaig adonar que allò no tenia sentit perquè és molt enriquidor ser de dos llocs.» (Gutiérrez 2013)

Así mismo, Karrouch y El Hachmi desmontan el decimonónico estereotipo «un individuo, una lengua, una cultura» para defender una idea más acorde con la sociedad europea contemporánea, multicultural y plurilingüe: «Jo parlo quatre llengües, tinc quatre cultures -l'àrab, la berber, la catalana i la castellana-, i això és una riquesa impressionant.» (Gutiérrez 2013) Es decir, frente a los prejuicios, la obra de estas dos autoras tan catalanas como marroquíes deja clara la idea de que el plurilingüismo suma, no divide.

Acabaremos estas páginas con palabras escritas por Laila Karrouch en Petjades de Nador, cuando debe dar una charla para alumnos de un instituto de secundaria. Una charla que la narradora describe como «corta y provechosa» al final de la cual los alumnos, al principio tímidos, se lanzan a hacerle preguntas sobre su vida entre dos culturas, dos países y varias lenguas: 
—Què t'estimes més, el Marroc o Catalunya?

Amb la mà encara alçada, m'hi vaig apropar i el vaig mirar de fit a fit.

-I tu qui t'estimes més el pare o la mare?

No va dubtar gens ni mica.

-A tots dos igual.

— Ja tens la resposta a la teva pregunta. (Karrouch 2013: Cap. V)

\section{BIBLIOGRAFÍA}

Agència Catalana de Notícies (4/7/2011) Najat El Hachmi: «Quan diuen 'nosaltres primer', qui és 'nosaltres'?». Nació Digital. Extraído de http://www. naciodigital.cat/noticia/33158/najat/hachmi/quan/diuen/nosaltres/primer/ qui/nosaltres

Alfaro, M. y Mangada, B. (2014) Atlas literario intercultural. Xenografías femeninas en Europa, Madrid, Calambur.

Bacardit, J. (4/6/2015) Najat El Hachmi: «Em sembla molt negatiu que per mostrar dubtes sobre el procés sobiranista siguis llençat a la foguera». Diari Crític. Extraído de http://www.elcritic.cat/entrevistes/najat-el-hachmitothom-esta-sorpres-que-jo-escrigui-sobre-sexe-per-que-perque-soc-marroquina-3627

Candel, F. (1974, 2008) Els altres catalans. Barcelona, Edicions 62.

El Hachmi, N. (2004) Jo també sóc catalana, Barcelona, Columna.

- (2008) L’últim patriarca, Barcelona, Planeta.

- (2015) La filla estrangera, Barcelona, Edicions 62.

Ferrer, Bernat (16/11/2013) Laila Karrouch: «Si Anglada diu que ens traiem el mocador, en portaré dos.» Nació Digital. Extraído de http://www.naciodigital.cat/noticia/61439/laila/karrouch/si/anglada/diu/traiem/mocador/portar/ dos

Gutiérrez, Thaïs (6/8/2013) Laila Karrouch: «És difícil ser dona i musulmana a Catalunya si tens un caràcter dèbil». Ara. Extraído de http://www.ara. cat/premium/opinio/LAILA-KARROUCH-musulmana-Catalunya-caracter_0_969503066.html

Karrouch, L. (2004) De Nador a Vic, Barcelona, Columna.

- (2013) Petjades de Nador, Barcelona, Columna. 
